ISSN 0719-7160

\title{
LUGARES SAGRADOS, RELIGIONES INDÍGENAS Y PATRIMONIO CULTURAL: EL CASO DEL CERRO COLO-COLO
}

\author{
[Sacred places, indigenous religions and cultural patrimony: the Cerro Colo-Colo \\ case]
}

\author{
CAROLINA SÁNCHEZ DE JAEGHER ${ }^{1}$ \\ RODRIGO CESPEDES ${ }^{2}$
}

Roa Antileo v Local Government of Arauco

Writ of protection, Case No. 289-2010 (10.08.2010), Court of Appeal of Concepcion (Chile)

Roa Antileo con Municipalidad de Arauco

Recurso de protección, Rol No. 289-2010 (10.08.2010), Corte of Apelaciones de Concepción

(Chile)

\begin{abstract}
The Colo-Colo hill, a sacred Mapuche place, suffered severe damages as a result of the 2010 Chilean Earthquake. The Municipality of Arauco undertook works to prevent landslides. By doing so, it cut sacred trees and removed some archaeological remains. The Municipality acted without prior consultation to the indigenous communities. The Chilcoco Indigenous Community brought the case to court for breaching the ILO Convention 169. The Appeal Court of Concepción ruled the measure was illegal and arbitrary, since these sacred sites cannot be intervened without prior consultation with indigenous communities, according to ILO Convention 169.
\end{abstract}

Key words: sacred places, cultural patrimony, indigenous rights, indigenous religions, ILO Convention 169

\begin{abstract}
Resumen
El cerro Colo-Colo, un lugar sagrado mapuche, sufrió severos daños a consecuencia del terremoto del 2010 y el Municipio de Arauco realizó labores tendientes a prevenir derrumbes, talando árboles sagrados y removiendo restos arqueológicos. La Municipalidad actuó sin previa consulta a las comunidades indígenas. La Comunidad Indígena Chilcoco, recurrió de protección frente a la omisión de un trámite esencial ordenada por un tratado de derechos humanos ratificado por Chile. La Corte de Apelaciones de Concepción calificó como arbitraria e ilegal la medida, pues los sitios sagrados no pueden ser intervenidos sin previa consulta a las comunidades indígenas según la Convención OIT 169.

Palabras clave: lugares sagrados, patrimonio cultural, derechos indígenas, religiones indígenas, Convención OIT 169
\end{abstract}

DOI: $10.7764 / R L D R .7 .87$

\footnotetext{
${ }^{1}$ Assistant Professor, University College Roosevelt - Utrecht University, Latin American Studies \& Southern Epistemologies. Email: c.sanchez@ucr.nl

${ }^{2}$ Senior research fellow, Max Planck Institute for Social Anthropology (Halle/Saale), Department of Law \& Anthropology. Email: cespedes@eth.mpg.de
} 
Carolina Sánchez De Jaegher y Rodrigo Cespedes: Lugares sagrados, religiones indígenas y patrimonio cultural: el caso del Cerro Colo-Colo

\section{Hechos}

El gran terremoto chileno del 2010 ocasionó graves deterioros en los cerros, ocasionando fisuras y derrumbes en los cerros. El cerro Colo-Colo, un lugar sacro mapuche, sufrió severos daños y, a raíz de esto, el Municipio de Arauco realizó labores tendientes a prevenir derrumbes. En esta acción preventiva, se talaron árboles sagrados y removieron restos arqueológicos produciendo perjuicios profundos al lugar sacro. La Municipalidad actuó sin previa consulta a las comunidades indígenas. Frente a esta situación, la Comunidad Indígena Chilcoco, a través de su Lonco, interpuso un recurso de protección ante la Corte de Apelaciones de Concepción, alegando que la Municipalidad había infringido el Convenio OIT 169 al no realizar los trabajos con previa consulta. Este hecho afectó sus derechos constitucionales consagrados en los Artículos 19 Nos. 1, 2, 6, 8 y 24 de la Carta Fundamental. Los recurrentes señalan además que los trabajos de la municipalidad provocaron destrucción de un sitio sagrado, con relevancia histórica y que es monumento nacional importante para su etnia. Además, alegaron que este sitio era esencial para las tradiciones y ceremonias propias de la cosmovisión indígena mapuche. Argumentaron que su integridad física y síquica fue afectada así como el ambiente, y la Municipalidad puso en un lugar secundario la libertad religiosa y el ejercicio del culto mapuche.

La Municipalidad informó que días después del terremoto, y como medida de seguridad por el riesgo que presentaban los árboles, se efectuaron trabajos por una empresa forestal para impedir el peligro de accidentes (posteriormente otra empresa explotó el bosque). Había entonces que evitar un peligro inminente de deslizamiento de tierra, para lo cual la Municipalidad de Arauco se vio en la necesidad de intervenir el Cerro Colo-Colo, mediante la realización de corte y extracción de árboles. Esto justificaba plenamente las medidas (y la omisión de la consulta) porque había una necesidad apremiante de intervenir el lugar para evitar accidentes potenciales. Entonces, debido a la urgencia, no se consultó a los pueblos indígenas por "la falta de organización y el elevado número de comunidades hacía dificultosa la comunicación y el consenso".

\section{Fallo}

La Corte de Apelaciones de Concepción, acogió el recurso de protección presentado por la comunidad mapuche Chilcoco en contra de la Municipalidad de Arauco. El Tribunal sostuvo que la Municipalidad violó el Convenio OIT 169, Artículos 6 y 13 y, consecuencialmente, vulneró el derecho a la integridad síquica de los recurrentes, garantía constitucional consagrada en el Artículo 19 No. 1. La Corte ordenó consultar a la Comunidad Indígena recurrente antes de reiniciar de nuevo los trabajos (la Municipalidad los detuvo al ser notificado el recurso).

La intervención municipal se realizó en un lugar declarado monumento histórico de especial importancia en el desarrollo de su culto y tradiciones propias de la etnia mapuche (hecho no controvertido). La importancia histórica del lugar era evidente ya que la misma Municipalidad solicitó y obtuvo la concesión de uso gratuito del cerro, con la finalidad de 
reconocer y reivindicar la cultura mapuche y establecer un desarrollo turístico étnico (una suerte de doctrina de los actos propios).

El Municipio realizó necesarias labores de mantención del lugar por fallas geológicas producidas por el gran terremoto del 27 de febrero de 2010. Sin embargo, las obras se realizaron sin la consulta previa a las comunidades indígenas, lo que hizo antijurídico su proceder ya que violó el Convenio OIT 169. En efecto, el Artículo 13 del Convenio dispone que los gobiernos deben respetar la importancia especial que reviste la relación espiritual que las comunidades indígenas con las tierras y territorios que ocupan o utilizan de alguna otra manera. Además, su Artículo 6 exige a los Estados consultar a los pueblos indígenas, mediante procedimientos apropiados $y$, en particular, a través de instituciones representativas cada vez que se prevean medidas susceptibles de afectarles directamente. Durante las labores de prevención se talaron indiscriminadamente árboles sagrados y removieron restos arqueológicos, lo que afectó directamente a las comunidades indígenas. La Corte remarcó que la destrucción del lugar sagrado que produjo un profundo impacto psicológico en las comunidades indígenas, dado que el cerro Colo-Colo había sido recientemente recuperado por estas comunidades tras años de lucha. El Municipio adujo que el hecho de no haber consultado a las comunidades se había hecho pensando en el tiempo y la dificultad de llegar a un acuerdo con la gran cantidad de comunidades mapuches afectadas. Esta excusa, según la Corte, no fue legítima ya que el tenor la Convención OIT 169 es imperativo. Además, antes de proceder a los trabajos, se hicieron diligencias y pidieron informes. Esto significaba que había un tiempo razonable para consultar a las comunidades indígenas. Esta circunstancia entonces prueba que la omisión de la consulta fue arbitraria.

En opinión de la Corte, los trabajos eran pertinentes, pero sólo debían ser realizados después de consultar a las comunidades, como lo señala el Convenio. La omisión de la consulta no puede justificarse en el elevado número de comunidades a consultar ni en la dificultad de un consenso. Siempre existe, según el Tribunal, la posibilidad de compatibilizar la seguridad pública y los intereses de las comunidades indígenas por sus lugares sagrados. La Corte remarca que "no cabe duda que la intervención y destrucción de su patrimonio cultural conduce a una sensación de falta de respeto a su identidad social, a sus costumbres y tradiciones, así como a la conservación de las características propias de su etnia, produciendo naturalmente desazón y gran preocupación" en la comunidad.

\section{Comentario}

Este fallo relevante es uno de los más relevantes en materia de religiones indígenas en Chile. $^{3}$ En primer lugar, la Corte considera como infringido el Artículo 13 de la Convención OIT $169,{ }^{4}$ que se reviere particularmente a lugares sagrados (en conjunción con su Artículo 6, ${ }^{5}$ sobre

\footnotetext{
3 En general, puede verse CÉSPEDES, Rodrigo, "Religiones Indígenas y Jurisprudencia Chilena", Revista Latinoamericana de Derecho y Religión 1.4 (2018).

4 “1. Al aplicar las disposiciones de esta parte del Convenio, los gobiernos deberán respetar la importancia especial que para las culturas y valores espirituales de los pueblos interesados reviste su relación con las tierras o
} 
Carolina Sánchez De Jaegher y Rodrigo Cespedes: Lugares sagrados, religiones indígenas y patrimonio cultural: el caso del Cerro Colo-Colo

consulta previa). ${ }^{6}$ Además, la Corte estima como infringido el Artículo 19 No. 1 de la Carta Fundamental Chilena, es decir el derecho a la vida e integridad física y psíquica, algo inusual en este tipo de casos. Este fundamento demuestra la importancia que otorga el Tribunal al lugar sagrado mapuche y el efecto que produjo su destrucción por la Municipalidad. La sentencia confirma a su vez la tendencia de considerar la consulta previa como un trámite esencia a toda medida o normativa que pueda afectar a los pueblos indígenas. Al mismo tiempo, la sentencia parece otorgar a la práctica de rituales ancestrales en lugares sagrados (y el atentado a la integridad psíquica por su destrucción) un sabor a derecho colectivo. ${ }^{7}$

En la última década y sobre todo en los países del cono sur, hemos visto la emergencia de la defensa de sus territorios ligada a la reivindicación de su patrimonio cultural indígena. En el caso del sitio ceremonial mapuche en el cerro Colo-Colo se trata también de esta emergencia que se suma a un sinnúmero de casos que están ocurriendo en Chile y Argentina que tienen que ver directamente con la defensa del territorio y, muy importante, con la forma de habitar en él. Es en este contexto que cuando la defensa del patrimonio cultural emerge en conjunto con la demandas de autonomía y territorio en el ámbito de la consulta, las comunidades están ejerciendo un derecho básico que no es correctamente traducible sólo a la conservación de sus sitios sagrados, sino más propiamente dicho tiene que ver con el derecho a existir y continuar haciéndose en y con estos espacios. Algo que, por cierto, aparece muy bien globalizado pero sin especificar en el Convenio 169 de la OIT en sus diferentes incisos relacionados a la protección de los de los derechos de los pueblos tribales.

En el contexto del conflicto Mapuche en el sur de Chile la necesidad de conservar los sitios sagrados está directamente ligada a la amenaza de exterminio que se vive hoy en todas las comunidades mapuches, puesto que sin territorio, tal como lo han venido señalando varios académicos y autoridades ancestrales mapuches, se deja de existir. En el fallo del cerro ColoColo, esto se ve muy bien ejemplificado. Dado que las raíces del litigio no se pueden entender completamente dentro de la perspectiva conservacionista, aunque el Convenio y también el fallo de este caso así lo refieran, la conservación de los espacios de carácter ancestral se trata de mucho más. Entendido desde la perspectiva indígena, el litigio por la preservación del sitio sagrado en el cerro Colo-Colo se refiere al ejercicio de una cosmovisión no-antropocéntrica. Recordemos que para los mundos relacionales las divisiones entre naturaleza y ser humano son muy porosas, a menudo inexistentes. ${ }^{8}$ La cosmovisión mapuche comprende la naturaleza como

territorios, o con ambos, según los casos, que ocupan o utilizan de alguna otra manera, y en particular los aspectos colectivos de esa relación.// 2. La utilización del término "tierras" en los artículos 15 y 16 deberá incluir el concepto de territorios, lo que cubre la totalidad del hábitat de las regiones que los pueblos interesados ocupan o utilizan de alguna otra manera." Sobre la diferencia entre "tierras" y "territorio" puede verse Agua Mineral Chusmiza SAIC v Comunidad Indígena Aymará de Chusmiza y Usmagana, Rol 2/840-2008, Corte Suprema, Casación, en CÉSPEDES, Rodrigo, Oxford Reports on International Law in Domestic Courts ILDC 1881 (CL 2009).

${ }^{5}$ Pueden verse también los Artículos 7.3 y 15.2 de la Convención OIT 169.

6 Relacionado con el tema de lugares sagrados, ver Artículos 11, 12 y 24 de la Declaración sobre los Derechos de los Pueblos Indígenas de la ONU (2007).

${ }^{7}$ En materia de lugares indígenas, puede verse también Comunidad Indígena Antú Lafquén de Huentetique con Corema de la Región de los Lagos (2012), Rol 10.090-2011, Corte Suprema.

8 Los pueblos indígenas canadienses tienen un concepto para describir esta noción: Wahkohtowin. Este término explica el entrelazamiento de la naturaleza y sus comunidades y los individuos que forman parte de esos grupos, que están en una especie de conexión simbiótica. En cierto sentido, se puede traducir como "derecho", no 
un ser viviente con seres que poseen gñen y newen. De ahí que para las prácticas ancestrales de espiritualidad mapuche tales como el We tripantu (inicio de la renovación del año mapuche mal traducido como año nuevo) se requiere el entorno con todos los gñen (espíritus) y newen (energías) que son dueños de estos espacios. Es por eso que para la comunidades indígenas alrededor del cerro Colo-Colo, la tala del bosque nativo haya sido percibida como una transgresión de su espiritualidad y una amenaza a su existencia como mapuche. En este sentido no se trata tan sólo de su integridad psíquica sino también de la posibilidad de que la identidad mapuche desaparezca en el torrente de la identidad estado-nación al no tener espacios en donde continuar su espiritualidad.

Se entiende entonces en este comentario que el patrimonio cultural en el marco de las cosmologías indígenas es mucho que la preservación física de espacios u objetos. ${ }^{9}$ La preservación de estos lugares se trata, en el sentido puramente espiritual mapuche, de seguir practicando aquellos rituales de reciprocidad con la madre tierra o Nuque Mapu de manera que ella siga proveyendo con la vida a todos sus seres. Sin estos actos de reciprocidad y complementación con la madre tierra, la continuación de la vida en comunidad y como mapuche se ve afectada y también detenida. Y esto se relaciona con el "Buen Vivir" mapuche o Küme

como reglas jerárquicas y formales a la manera occidental, sino como una serie de obligaciones personales hacia otros individuos, la tribu y la naturaleza en su conjunto, que es considerada como una unidad. De esa manera, la identidad personal es inseparable de la tierra, las plantas, los animales y los grupos humanos. Esos deberes no son sólo son obligaciones legales en el sentido occidental, sino también religiosas, deberes trascendentes que tienen fundamentos sagrados. Esa base sacra proviene de una observación colectiva de los ciclos naturales y es transmitida por la tradición oral. De eso, los pueblos indígenas derivaron una especie de sentido religioso/moral/legal, que incluye compasión con respecto a otras personas, pero también otras criaturas y el ambiente en sí (Pachamama) que es una entidad separada, pero en estrecha relación con Las comunidades humanas, que también forman parte del sistema. El sentido de justicia de los pueblos indígenas y su noción de derechos se deriva del mismo punto de vista. Entonces, la justicia es una especie de equilibrio cósmico y los derechos legales están informados con el concepto de "buen vivir" o "vivir bien". Ambas concepciones, justicia y derechos, han desempeñado un papel en fallos dictados por los tribunales. Ver BUHLER, Sarah, "Reading Law and Imagining Justice in the Wahkohtowin Classroom", Windsor Yearbook of Access to Justice/Recueil Annuel de Windsor d'accès à la Justice 34.1 (2017), pp 175-188; y NAPOLEON, Val, "Thinking about Indigenous legal orders", Dialogues on Human Rights and Legal Pluralism, Springer, Dordrecht, 2013, pp 229-245.

${ }^{9}$ El patrimonio cultural (tangible o intangible) es la herencia cultural propia del pasado de un grupo humano, mantenida hasta la actualidad y transmitida a las generaciones presentes y futuras (ver la Convención sobre la Protección del Patrimonio Mundial Cultural y Natural de la UNESCO de 1972). En el caso de los indígenas, la Declaración de las Naciones Unidas sobre los Derechos de los Pueblos Indígenas de la ONU (2007), que señala que los pueblos indígenas tienen derecho a mantener, controlar, proteger y desarrollar su patrimonio cultural, a practicar y revitalizar sus tradiciones y costumbres; a practicar y enseñar sus ceremonias espirituales; a mantener y proteger sus lugares religiosos y culturales y a acceder a ellos; a mantener su identidad étnica, así como a la restitución de los bienes culturales y espirituales de los cuales hayan sido despojados. En este caso también es atinente la Convención para la Salvaguarda del Patrimonio Cultural Inmaterial de la UNESCO (2003). El Capítulo IV, Artículos 30-32 de las Constitución Boliviana incluye los derechos de identidad cultural, libertad religiosa, títulos de tierra, protección de lugares sagrados, etc. La American Indian Religious Freedom Act (1978) que les otorgó a los indígenas su derecho a ejercitar sus prácticas tradicionales (de los cuales fueron despojados). Estas libertades comprenden el acceso a lugares sagrados y la libertad de practicar rituales tradicionales (que incluyan drogas como el peyote). Sobre el tema puede verse, KUPPE, René, "Religious freedom law and the protection of sacred sites", in Permutations of Order: Religion and Law as Contested Sovereignties, Routledge, 2016, pp 63-80. 
Carolina Sánchez De Jaegher y Rodrigo Cespedes: Lugares sagrados, religiones indígenas y patrimonio cultural: el caso del Cerro Colo-Colo

Mogen que incluye principios como el itxofill mogen (una idea cercana, aunque no del todo traducible como "biodiversidad"), que indica dentro de la normativa indígena que hay que vivir con la naturaleza y no vivir solamente de ella y sus recursos naturales.

Los rituales mapuches de complementación con la madre tierra exigen también la reserva de estos espacios como intocables por los seres humanos; es decir, en estos espacios donde el che (persona) devuelve ceremonialmente lo que ha tomado de la madre tierra. Propiamente hablando, no son lugares de patrimonio cultural como lo entenderíamos en Occidente sino que, desde la mirada indígena, son espacios que ya tienen dueños y ellos son los gñen y newen particulares de cada uno de estos lugares. La labor del che (persona) es entonces ser una especie de guardián, cuya misión es que no se quebrante el equilibrio establecido en los lugares sagrados $y$, de esta manera, la madre tierra pueda seguir sosteniéndonos en equilibrio con todos los otros seres: el ser árbol, el ser río, el ser montaña, en fin todas las existencias del territorio. Es en este punto en donde la tala de un árbol o bosques antiguos en territorio indígena y, especialmente en estos espacios sagrados, no debe entenderse solamente desde el punto de vista de preservación de monumentos o de la biodiversidad. ${ }^{10}$

La consulta previa ha establecido uno de lo más potentes instrumentos legales por medio de la cual las comunidades mapuches han podido frenar, y muchas veces salvaguardar espacios, que son vitales para su supervivencia. Sin embargo, y tal como lo deja ver la descripción del fallo del cerro Colo-Colo, la aplicación efectiva de esta normativa exigida en el Convenio 169 de la OIT, va ligada al conocimiento que tengan las autoridades nacionales sobre la organización política de las comunidades mapuche. De ahí, que su desconocimiento dificulta la ejecución y desenvolvimiento de la consulta, puesto que muchas veces estas autoridades chocan con la organización mapuche que desconoce la jerarquía de vertical occidental desde "arriba-abajo".

En primer lugar hay que tener en cuenta que se ha señalado la dificultad de establecer diálogos con las comunidades mapuches debido a la carencia de líderes con quién se pueda hablar directamente. En nuestra opinión, en esta dificultad juega un rol importante el desconocimiento e incertidumbre de cómo entablar el acercamiento a las comunidades que se deriva, principalmente, de la falta de tradición en establecer diálogos horizontales con las comunidades, principio que choca directamente con la jerarquía vertical de poder desde la cúpula "hacia abajo". La horizontalidad, a la hora de tomar acuerdos entre comunidades es una noción que las comunidades mapuches sí poseen en su modo ancestral de hacer política. En la práctica, esto se traduce en que en la toma de decisiones, la organización política mapuche recurre al trawün (que son encuentros en donde se delibera con los loncos y machis, autoridades política de alto rango, las decisiones con los miembros de la comunidad). Cuando hay más de una comunidad envuelta en la decisión de algo relevante se llama a un futra-trawün, que incluye a todas esas comunidades con sus loncos y autoridades correspondientes. De manera que, para organizar una consulta con las comunidades mapuches, hay que establecer primero protocolos con la base consultiva de los trawün que son la forma política deliberativa que tienen las comunidades.

${ }^{10}$ La Corte Inter-Americana de Derecho Humanos ha reconocido esta especial relación espiritual entre los indígenas y la tierra; por ejemplo, en Mayagna (Sumo) Awas Tingni Community v Nicaragua (2001) y en Comunidad Indígena Yakye Axa v Paraguay (2005). En forma similar, el Comité de Derecho Humanos de la ONU ha reconocido esta especial conexión en Hopu \& Bessert v France (1997), Communication No. 549/1993, UN Doc. CCPR/C/60/D/549/1993/Rev.1. 
ISSN 0719-7160

Es importante recordar que la alusión al tiempo descrita en la defensa de las labores efectuadas por la Municipalidad, no tiene nada que ver con la dilatación de la consulta y la imposibilidad de llegar a un acuerdo, situación que puede pasar en diferentes espacios no indígenas también debido a la diferencia de intereses. Creemos además que para la realización de un contexto propicio que lleve a cabo la consulta indígena más expeditamente, es imprescindible que se establezcan puentes de entendimiento entre las diferentes formas de deliberación. 